\title{
LEVANTAMENTO FITOSSOCIOLÓGICO DE PLANTAS DANINHAS EM CAFEZAL ORGÂNICO(')
}

\author{
CLEBER DANIEL DE GOES MACIEL $\left({ }^{2 *}\right)$; JULIANA PARISOTTO POLETINE $\left({ }^{2}\right)$; \\ ANTÔNIO MENDES DE OLIVEIRA NETO ( $\left.{ }^{3}\right)$; NAIARA GUERRA $\left({ }^{3}\right)$; WAGNER JUSTINIANO ( ${ }^{(3)}$
}

\begin{abstract}
RESUMO
A adequação de um cafezal para o sistema orgânico causa grande mudança no sistema de manejo de plantas daninhas, mas as informações sobre o comportamento da comunidade infestante em áreas de cultivo de café orgânico são escassas no Brasil. Objetivou-se com este trabalho realizar um levantamento fitossociológico da comunidade infestante em três cafezais (variedades Mundo Novo, Bourbon Vermelho e Obatã) desenvolvidos em sistema orgânico, no município de Garça (SP). Nas três áreas estudadas foram arremessados ao acaso cem quadrados metálicos com área vazada de $0,25 \mathrm{~m}^{2}$, nas entrelinhas da cultura. As espécies contidas no interior dos quadros foram identificadas segundo a espécie botânica, nome popular e família. A partir dos resultados, determinaramse os parâmetros fitossociológicos: frequência, densidade, abundância, frequência relativa, densidade relativa, abundância relativa, índice de valor de importância e similaridade florísticas pelos métodos de Simple Matching de Sneath \& Sokal e similaridade de Sorensen. As famílias que mais se destacaram, com maior número de espécies nas três áreas foram Poaceae e Asteraceae. Houve alta similaridade em todos os contrastes testados, já que pelo menos metade das espécies identificadas foi comum às duas áreas contrastadas.
\end{abstract}

Palavras-chave: fitossociologia, Coffea arabica, cultivares, agricultura orgânica.

\section{ABSTRACT \\ WEEDS PHYTOSOCIOLOGICAL SURVEY IN ORGANIC COFFEE CROP}

Adjust of one coffee crop to organic system causes significative changes in weed management system, but information about the weed community in areas cultivated with organic coffee crop are scarce in Brazil. This work aimed to perform a phytosociological survey of weed community in three coffee crop areas (Mundo Novo, Bourbon Vermelho and Obatã genotypes) conducted in organic system, in Garça County, São Paulo State. In the three analyzed areas, a hundred of metallic drained squares were randomized hurled with useful area of $0.25 \mathrm{~m}^{2}$ between crop lines. Plants contained in the interior of squares were identified according to botanic species, common name and its family. Phytosociological parameters were determined: frequency, density, abundance, relative frequency, relative density, relative abundance, index of importance value, and floristic similarity by Simple Matching of Sneath \& Sokal and similarity by Sorensen method. Outstanding families, with major species number in the three studied areas were Poaceae and Asteraceae. There was high similarity in all studied contrasts, since at least a half of identified species was common to the two contrasted areas.

Key words: phytosociology, Coffea arabica, cultivars, organic agriculture.

(1) Recebido para publicação em 28 de agosto de 2009 e aceito em 25 de fevereiro de 2010.

(2) Escola Superior de Agronomia de Paraguaçu Paulista (ESAPP/FUNGE), Departamento de Fitotecnia, Caixa Postal 88, 19700-000 Paraguaçu Paulista (SP). E-mail: jupoletine@hotmail.com; cleber_goes_maciel@hotmail.com $\left(^{*}\right)$ Autor correspondente.

(3) Escola Superior de Agronomia de Paraguaçu Paulista (ESAPP/FUNGE), Acadêmicos do Curso de Agronomia. Paraguaçu Paulista (SP). E-mail: am.oliveiraneto@hotmail.com; naiara.guerra@hotmail.com; wanagro@hotmail.com 
O Brasil é o maior e mais tradicional produtor mundial de café, produto que atualmente envolve cerca de 10 milhões de pessoas em sua cadeia produtiva. Em paralelo, a cafeicultura orgânica vem crescendo a elevadas taxas anuais, e apesar de ainda representar pequena fatia do mercado total, tem enorme potencial de crescimento devido ao seu apelo ambiental, social e econômico, tornando-se excelente opção para pequenos e médios agricultores (RICCI et al., 2006).

As plantas daninhas competem pelos mesmos recursos exigidos pelo cafeeiro e, quando ambas se desenvolvem no mesmo ambiente, há redução significativa na produção de grãos (ToLEDO et al., 1996). Portanto, a observação sobre as espécies de plantas daninhas que ocorrem na lavoura cafeeira é importante para a escolha mais adequada do método de controle, uma vez que pode influir diretamente na eficiência do manejo a ser utilizado.

Segundo relatos de Ronchi et al. (2003; 2007), Ronchi e SiLva (2006) plantas daninhas como Bidens pilosa, Commelina diffusa, Leonurus sibiricus e Richardia brasiliensis, Sida rhombifolia e Nicandra physaloides, mesmo sob baixa infestação, podem reduzir significativamente o acúmulo de nutrientes de plantas jovens de café, assim como o diâmetro do caule, número de folhas e massa seca da parte aérea.

Para a agricultura orgânica, as plantas daninhas, consideradas plantas espontâneas nos sistemas de café orgânico, têm importância por serem componentes de: reciclagem de nutrientes das camadas mais profundas do solo; promoção da descompactação, assim como proteção contra erosão e incidência direta de radiação solar, a redução da amplitude térmica, manutenção da matéria orgânica e aeração no solo, assim como do estabelecimento de condições favoráveis ao controle biológico de pragas através da diversidade de espécies (RICCI et al., 2002; Azevedo et al., 2002).

Ricci et al. (2008) descreveram aumento da frequência de espécies de plantas daninhas no sistema orgânico com média e baixa utilização de insumos, ao contrário do sistema convencional, com alto ou médio consumode insumose aplicação do herbicida glyphosate, onde houve aumento de gramíneas que são, na maioria dos casos, indesejáveis em um manejo seletivo.

O objetivo desse trabalho foi realizar um levantamento fitossociológico da comunidade infestante em cafezais das variedades Mundo Novo, Bourbon Vermelho e Obatã. Levantamentos fitossociológicos em três localidades produtoras de café cultivado no sistema orgânico foram realizados em março e abril de 2005, em propriedades rurais localizadas no município de Garça (SP).

As áreas do estudo foram representadas por: Área 1 e 2, pertencentes ao Sítio São José, cultivadas desde 2001 e 2002, respectivamente, no sistema orgânico, as variedades Mundo Novo e Bourbon Vermelho e Área 3, pertencente ao Sítio Águas Claras, cultivada no sistema orgânico a variedade Obatã desde 2003. Nessas áreas, o solo é classificado como Latossolo Vermelhodistroférrico, textura arenosa, com relevo levemente ondulado. O clima da região é do tipo Aw, segundo classificação de Köppen (1948), sendo caracterizado por savana com temperatura média anual de $22,8{ }^{\circ} \mathrm{C}$ e amplitude em torno de $5{ }^{\circ} \mathrm{C}$. Registra precipitação pluvial média anual de $1.360 \mathrm{~mm}$, com período chuvoso de dezembro a fevereiro e o seco de junho a agosto. $\mathrm{Na}$ figura 1, verificam-se os dados climatológicos referentes ao período de execução do trabalho.

As amostragens foram tomadas arremessandose, aleatoriamente nas entrelinhas da cultura, cem

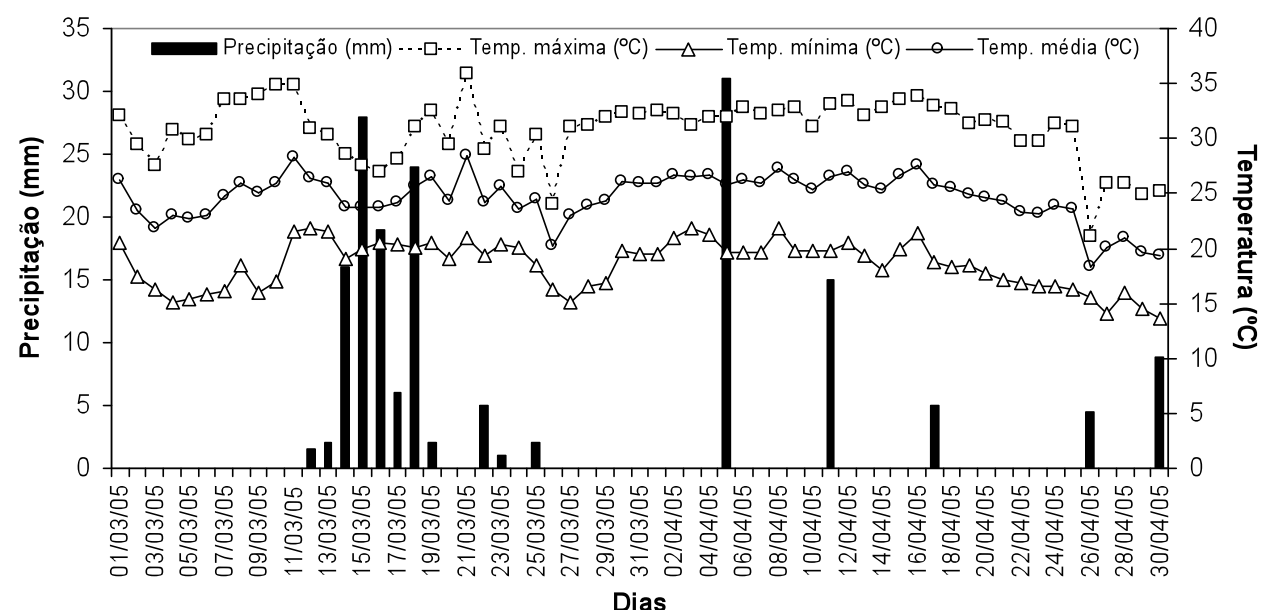

Figura 1. Variação diária da temperatura (máxima, mínima e média) e precipitação pluvial durante os levantamentos fitossociológicos em cafezal orgânico. Garça (SP). Fonte: CiIAGro (2009). 
quadrantes vazados com área interna de 0,25 $\mathrm{m}^{2}$, totalizando $25 \mathrm{~m}^{2}$ por área amostrada, semelhantemente à descrita por OLIVEIRA e FreITAS (2008). O Sistema de classificação adotado como base foi o sistema ANGIOSPERM ... (2003), com auxílio nas delimitações das famílias e ordenamento de alguns gêneros, em SouzA e LoRENZI (2005). Entretanto, na identificação e quantificação das plantas daninhas por nome científico, nome popular e família, também foi utilizada literatura específica semelhante à descrita por LORENZI (2008).

A partir da identificação das espécies, foram calculados os parâmetros fitossociológicos: número de indivíduos por espécie, número de parcelas em que a espécie estava presente, frequência (índice da ocorrência das espécies em cada quadrado), densidade (índice da quantidade de indivíduos de uma mesma espécie em cada quadrado), abundância (concentração das espécies nos diferentes pontos da área total), frequência relativa, densidade relativa e abundância relativa (relaciona uma espécie a todas as demais encontradas nas áreas) e o índice de valor de importância. No cálculo das variáveis, foram utilizadas as equações descritas a seguir (MUELLER-DOMBOIS e ELLENBERG (1974): Frequência $($ Fre $)=($ parcelas que contém a espécie $) /$ (total de parcelas utilizadas); Densidade (Den) $=($ total de indivíduos por espécie) / (área total coletada); Abundância (Abu)=(total de indivíduos por espécie)/(total de parcelas contendo a espécie); Frequência Relativa (Frr)=(frequência da espécie x 100)/(frequência total de todas as espécies); Densidade Relativa (Der) $=($ densidade da espécie $x$ 100) / (densidade total de todas as espécies); Abundância Relativa $(\mathrm{Abr})=($ abundância da espécie $x$ 100)/ (abundância total de todas as espécies); Índice de Valor de Importância (IVI) $=($ Frr + Der + Abr $)$.

Determinou-se o índice de similaridade florística por duas metodologias distintas, a primeira pelo método de Simple Matching de Sneath e Sokal (1973), citado por Nordi (1996), e a segunda, através do índice de similaridade de Sorensen, citado por OliveIRA e Freitas (2008).

No levantamento fitossociológico das áreas de cultivo de café orgânico do município de Garça (SP) foram encontradas 41 espécies de plantas espontâneas distribuídas em 18 famílias. A família com maior número de espécies foi a Poaceae, com um total de 12 espécies, seguida de Asteraceae, Euphorbiaceae, Convolvulaceae e Labiatae, com respectivamente 9, 3, 2 e 2 espécies (Tabela 1). Esses resultados corroboram com o verificado em levantamento realizado em consórcio entre café e grevíleas no município de Vitória da Conquista, BA (SILVA et al., 2006) e no sistema orgânico entre café e pupunha no município de Garça, SP (BARBOSA, 2004).

As espécies Panicum maximum, Portulaca oleracea, Emilia sonchifolia e Bidens pilosa, que foram identificadas no levantamento e se destacaram entre as principais (Tabela 1), também foram relatadas em levantamentos realizados nos municípios de São Sebastião do Paraíso (MG) e Vitória da Conquista (BA) na cultura do café convencional (AlCÂNTARA e FERREIRA, 2000; SILVA et al., 2006). PARTELLI (2008), avaliando plantas de cobertura do solo em sistema de café orgânico, relatou que as espécies Canavalia ensiformis, Mucuna deeringiana e Pennisetum glaucum promoveram modificações na dinâmica de sucessão de plantas espontâneas, auxiliando no controle e sem prejudicar o cafeeiro.

$\mathrm{Na}$ área cultivada com a variedade Obatã, registrou-se a maior diversidade de espécies, onde foram constatadas 38 das 41 espécies identificadas no conjunto dos três levantamentos. Esse valor superou as áreas cultivadas com Bourbon Vermelho e Mundo Novo, e identificadas 25 e 23 espécies do total respectivamente (Figura 2). Essa maior diversidade pode ser resultado da implantação mais recente daquela lavoura, onde pode ainda não ter havido influência na espontaneidade do sistema de manejo orgânico ou mesmo da variedade de café utilizada. Ricci et al. (2008), relataram decréscimo na diversidade de espécies com o aumento da idade do cafezal consorciado desenvolvido em manejo orgânico com médio e baixo consumo de insumos.

Na área da variedade Mundo Novo as famílias Poaceae e Asteraceae foram as de maior destaque registrando, respectivamente, 9 e 5 espécies do levantamento (Figura 2). Com relação ao índice de valor de importância, a C. rotundus $(53,95), R$. brasiliensis $(33,45)$, D. horizontalis $(28,69), G$. parviflora $(22,64), B$. pilosa $(22,41)$ e E. indica $(20,71)$ destacaram-se na área com a variedade Mundo Novo.

As famílias Poaceae e Asteraceae também se destacaram para a variedade Bourbon Vermelho, com a ocorrência de, respectivamente, 7 e 6 espécies do total (Figura 2). Em relação ao índice de valor de importância, destacaram-se C. rotundus (56,09), G. parviflora $(33,68)$, P. maximum $(27,68)$, B. pilosa $(23,70)$ e C. benghalensis $(19,23)$. Para as variedades Mundo Novo e Bourbon Vermelho, as espécies espontâneas $D$. horizontalis e P. maximum foram identificadas com elevada abundância e valor de importância, não corroborando com as informações relatadas por RICCI et al. (2008), em que os autores mencionam ser mais comum o aumento de espécies gramíneas em sistemas de cultivo convencional submetidos à aplicação de herbicidas.

$\mathrm{Na}$ área da variedade Obatã, foi observada a maior diversidade de espécies de plantas daninhas do levantamento, onde novamente as famílias Poaceae e Asteraceae se destacaram com o maior número de espécies (Figura 3). Destacaram-se como as espécies de 
Tabela 1. Relação de espécies, nomes populares e família de plantas espontâneas identificadas nos levantamentos realizados em cafezais (Coffea arabica L.) cultivados com as variedades Mundo Novo, Bourbon Vermelho e Obatã, no município de Garça (SP)

\begin{tabular}{|c|c|c|}
\hline Espécie & Nome Popular & Família \\
\hline Brachiaria decumbens & capim-brachiaria & Poaceae \\
\hline Brachiaria plantaginea & capim-marmelada & Poaceae \\
\hline Cenchrus echinatus & capim-carrapicho & Poaceae \\
\hline Cynodon dactylon & grama-seda & Poaceae \\
\hline Digitaria horizontalis & capim-colchão & Poaceae \\
\hline Digitaria insularis & capim-amargoso & Poaceae \\
\hline Eleusine indica & capim-pé-de-galinha & Poaceae \\
\hline Eragrostis pilosa & capim-mimoso & Poaceae \\
\hline Panicum maximum & capim-colonião & Poaceae \\
\hline Rhynchelytrum repens & capim-favorito & Poaceae \\
\hline Setaria geniculata & capim-rabo-de-raposa & Poaceae \\
\hline Acanthospermum australe & carrapichinho & Asteraceae \\
\hline Acanthospermum hispidum & carrapicho-de-carneiro & Asteraceae \\
\hline Ageratum conyzoides & picão-roxo & Asteraceae \\
\hline Bidens pilosa & picão-preto & Asteraceae \\
\hline Conyza bonariensis & buva & Asteraceae \\
\hline Emilia sonchifolia & falsa-serralha & Asteraceae \\
\hline Galinsoga parviflora & picão-branco & Asteraceae \\
\hline Gnaphalium spicatum & macela & Asteraceae \\
\hline Tridax procumbens & erva-de-touro & Asteraceae \\
\hline Chamaesyce hirta & erva-de-santa-luzia & Euphorbiaceae \\
\hline Euphorbia heterophylla & amendoim-bravo & Euphorbiaceae \\
\hline Ricinus communis & mamona & Euphorbiaceae \\
\hline Hypits lophantha & hortelã & Labiatae \\
\hline Marsypianthes chamaedrys & hortelã-do-campo & Labiatae \\
\hline Ipomoea grandifolia & corda-de-viola & Convolvulaceae \\
\hline Ipomoea quamoclit & corda-de-viola-esqueleto & Convolvulaceae \\
\hline Amaranthus retroflexus & caruru & Amarantaceae \\
\hline Lepidium virginicum & mentruz & Brassicaceae \\
\hline Spergula arvensis & maria-gorda & Caryophyllaceae \\
\hline Chenopodium ambrosioides & erva-de-santa-maria & Chenopodiaceae \\
\hline Commelina benghalensis & trapoeraba & Commelinaceae \\
\hline Momordica charantia & melão-de-são-caetano & Curcubitaceae \\
\hline Cyperus rotundus & tiririca & Cyperaeae \\
\hline Sida glaziovii & guanxuma-branca & Malvaceae \\
\hline Mollugo virticillata & capim-tapete & Molluginaceae \\
\hline Desmodium tortuosum & pega-pega & Papilionoidae \\
\hline Portulaca oleracea & beldroega & Portulacaceae \\
\hline Richardia brasiliensis & poaia-branca & Rubiaceae \\
\hline Solanum americanum & maria-pretinha & Solanaceae \\
\hline
\end{tabular}

maior importância B. pilosa $(44,90)$, C. rotundus $(40,96)$, C. benghalensis $(26,94)$ e E. sonchifolia $(20,98)$, diferentemente das variedades Mundo NovoeBourbon Vermelho, uma vez que não se constatou nenhuma espécie da família Poaceae entre as de maior IVI, apesar de ter sido identificado o maior número de espécies do levantamento.

Nas três áreas levantadas, foi comum a presença de $C$. rotundus e $B$. pilosa entre as espécies com maior frequência e maior IVI, assim como C. benghalensis foi comum para as variedades Bourbon Vermelho e Obatã, caracterizando resultados que corroboram com os verificados por Silva et al. (2006). CARvalHo et al. (2002) e Ricci et al. (2002), mencionaram os efeitos inibidores (alelopáticos) dos adubos verdes Crotalaria juncea, $C$. ensiformis, Stilozobium aterrimum e Mucuna pruriens sobre o desenvolvimento de C. rotundus e B. pilosa. As 


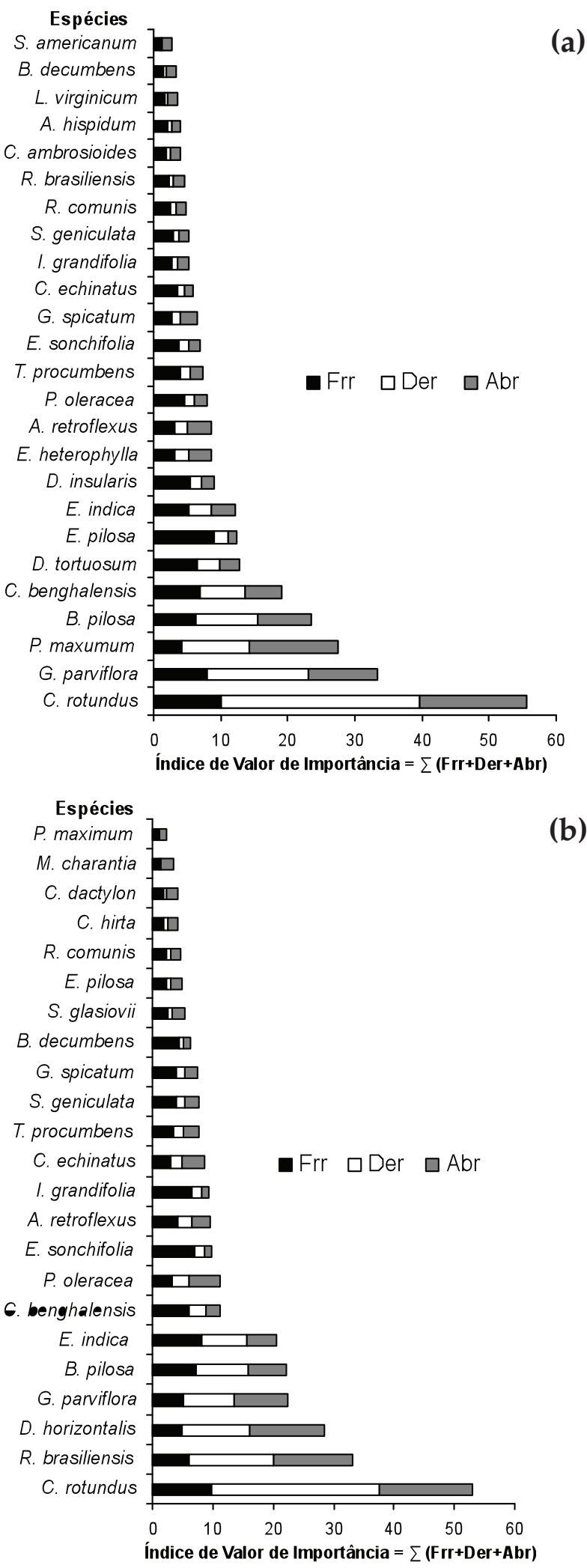

Figura 2. Índice de valor de importância caracterizado pelo somatório de porcentagem da frequência relativa (Frr), densidade relativa (Der) e abundância relativa (Abr) das espécies de plantas espontâneas constituintes de cafezal (Coffea arabica L.) variedades Mundo Novo (a) e Bourbon Vermelho (b), no município de Garça (SP).

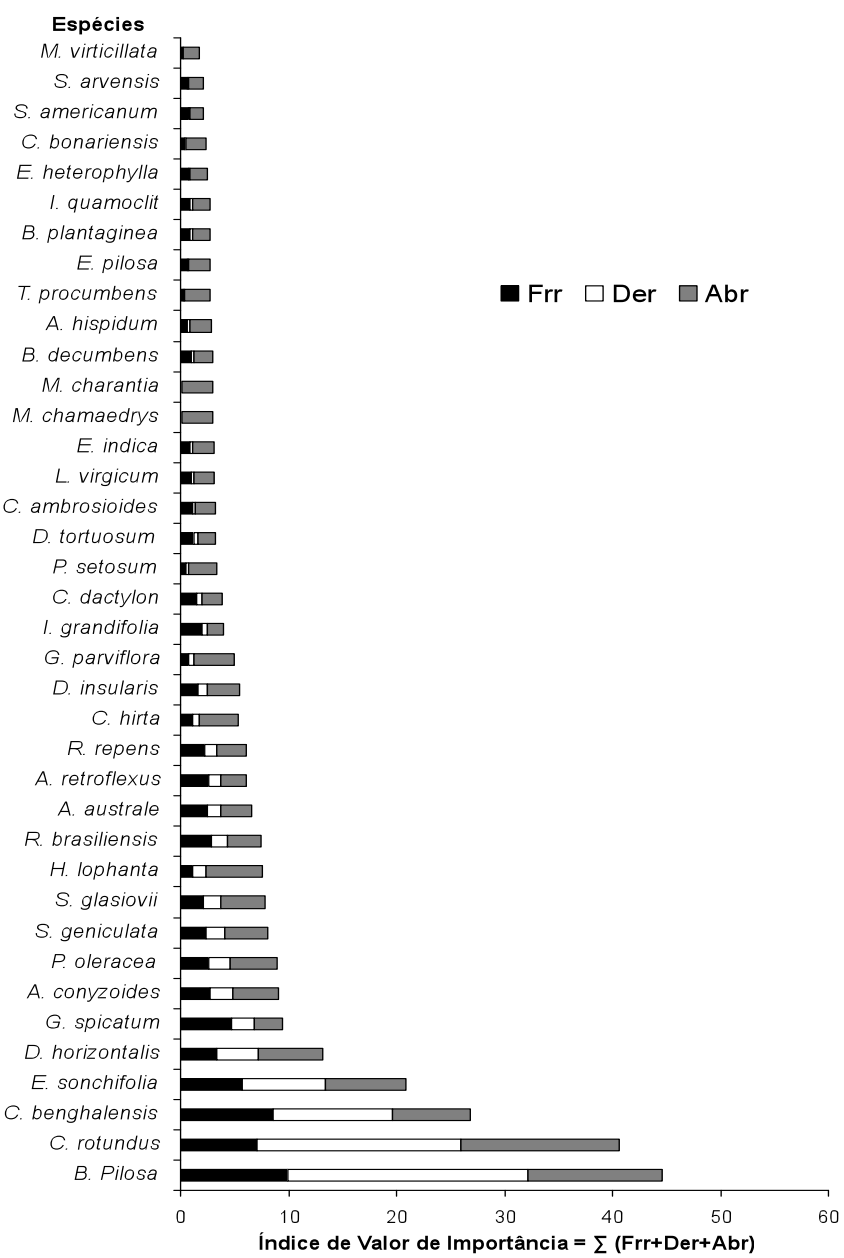

Figura 3. Índice de valor de importância caracterizado pela somatória de porcentagem da freqüência relativa (Frr), densidade relativa (Der) e abundância relativa (Abr) das espécies de plantas espontâneas constituintes de cafezal (Coffea arabica L.) variedade Obatã, no município de Garça (SP).

informações resultantes do levantamento florístico fornecem subsídios que indicam a viabilidade dos adubos verdes $C$. juncea, $C$. ensiformis, S. aterrimum e $M$. pruriens para a supressão das espécies espontâneas de maior IVI nas áreas de café orgânico avaliados.

Pelo índice de similaridade (IS\%) entre as variedades nota-se alta semelhança das espécies entre as áreas onde foram desenvolvidos os levantamentos. Todos os contrastes tiveram valores superiores a $50 \%$ para os índices percentuais de similaridade (IS\%) e 0,49 para similaridade florística (SM), demonstrando que pelo menos metade das espécies foi comum às áreas contrastadas.

É importante ressaltar que o conhecimento prévio da composição florística das plantas espontâneas em sistemas de café orgânico, permite antecipar a organização de estratégias preventivas para adoção 
de medidas de controle mais sustentáveis. Nesse contexto, como foram poucas as espécies dominantes nas diferentes localidades, seria possível adoção de medidas de controle em função das espécies de maior ocorrência, associando o uso de adubos verdes com roçadas ou capinas seletivas, e continuidade do monitoramento para se evitar o aumento das espécies de menor ocorrência.

Para as áreas de café cultivadas em sistema orgânico, verificou-se elevada similaridade florística das espécies de plantas espontâneas, ocorrendo maior número de espécies pertencentes às famílias Poaceae e Asteraceae. A partir da análise dos índices populacionais foi verificada grande expressão da espécie Cyperus rotundus para as áreas cultivadas com as variedades Mundo Novo, Bourbon Vermelho e Obatã. Apesar do manejo orgânico, para as áreas com as variedades Mundo Novo e Bourbon Vermelho, foi observada significativa interação populacional das espécies Panicum maximum e Digitaria horizontalis.

\section{REFERÊNCIAS}

ALCÂNTARA, E.N.; FERREIRA, M.M. Efeito de diferentes métodos de controle de plantas daninhas sobre a produção de cafeeiros instalados em Latossolo Roxo distrófico. Ciência e Agrotecnologia, v.4, p.54-61, 2000.

ANGIOSPERM PHYLOGENY GROUP - APG II. An update of the Angiosperm Phylogeny Group classification for the orders and families of flowering plants: APG II. Botanical Journal of the Linnean Society, n.141, p.399-436, 2003.

AZEVEDO, M.F.R.; LIMA P.C.; ESPINDOLA, J.A.A.; MOURA, W.M. Conversão de cafezais convencionais em orgânicos. Informe Agropecuário, v.23, p.53-61, 2002.

BARBOSA, M.R. Café e pupunha: um casamento perfeito. Agroecologia Hoje, n.23, p.6-7, 2004.

CARVALHO, F.S.; FONTANÉTTI, A.; SANTOS, C.T.C. Potencial alelopáticos do feijão-de-porco (Canavalia ensiformes) e da mucuna preta (Stilozobium aterrimum) no controle da tiririca (Cyperus rotundus). Ciência e Agrotecnologia, v.26, p.647-651, 2002.

KÖPPEN, W. Climatologia: con uno estudio de los climas de la tierra. México City: Fondo Cultura Econômica, 1948. 478p.

LORENZI, H. Plantas daninhas do Brasil: terrestres, aquáticas, parasitas e tóxicas. 4. ed. Nova Odessa: Plantarum, 2008. 640p.

MUELLER-DOMBOIS, D.; ELLENBERG, H. Aims and methods of vegetation ecology. New York: J. Wiley. 1974. 547p.
NORDI, J.C. Composição florística e estrutura da comunidade de plantas daninhas em cultura de Eucalyptus grandis W. Hell ex. maiden. 1996. 146f. Dissertação (Mestrado em Ciências Biológicas/Botânica) - Instituto de Biociências de Botucatu, Universidade Estadual Paulista, Botucatu.

OLIVEIRA, A.R.; FREITAS, S.P. Levantamento fitossociológico de plantas daninhas em áreas de produção de cana-de-açúcar. Planta Daninha, v.26, p.33-46, 2008.

PARTELLI, F.L. Aspectos microbiológicos, nutricionais, fisiológicos e bioquímicos em cafeeiro. 2008. 227f. Tese (Doutorado em Agronomia/Produção vegetal) - Universidade Estadual do Norte Fluminense Darcy Ribeiro, Rio de Janeiro.

RICCI, M.S.F.; ARAÚJO, M.C.F.; FRANCH, C.M.C. Cultivo orgânico do café: recomendações técnicas. Brasília: EMBRAPA Informação Tecnológica, 2002. 101p.

RICCI, M.S.F.; NEVES, M.C.P.; NANNETI, A.N.; MOREIRA, C.F.; AGUIAR-MENEZES, E.L.; SILVA, E.; CAIXETA, I.F.; ARAÚJO, J.B.S.; LEAL, M.A.A; FERNANDES, M.C.; ALMEIDA, P.S.; PEDINI, S. Cultivo do café orgânico. Sistemas de produção, 2 ed., Dez 2006. Disponível em:http:/ / www. sistemasdeproducao. cnptia.embrapa.br / fontesHTML/ cafe/ cafeorganico_2ed/autores.htm. Acesso em: 20 dez. 2008.

RICCI, M.S.F.; VÍRGILIO FILHO, E.M.; COSTA, J.R. Diversidade da comunidade de plantas invasoras em sistemas agroflorestais com café em Tuirrialba, Costa Rica. Pesquisa Agropecuária Brasileira, v.43, p.825-834, 2008.

RONCHI, C.P.; TERRA, A.A.; SILVA, A.A.; FERREIRA, L.R. Acúmulo de nutrientes pelo cafeeiro sob interferência de plantas daninhas. Planta Daninha, v.21, p.219-227, 2003.

RONCHI, C.P.;SILVA, A.A. Effects of weed species competition on the growth of young coffee plants. Planta Daninha, v.24, p.415-423, 2006.

RONCHI, C.P.; TERRA, A.A.; SILVA, A.A. Growth and nutrient concentracion in coffee root system under weed species competition. Planta Daninha, v.25, p.679-687, 2007.

SILVA, S.O.; MATSUMOTO, S.N.; BEBÉ, F.V.; JOSÉ, A.R.S. Diversidade e freqüência de plantas daninhas em associação entre cafeeiros e grevíleas. Coffee Science, v.1, p.126-134, 2006.

SOUZA, V.C.; LORENZI, H. Botânica sistemática: guia ilustrado para identificação das famílias de angiospermas da flora brasileira, baseado em APG II. 1.ed. Nova Odessa: Plantarum, 2005. 640p.

TOLEDO, S.V.; MORAES, M.V.; BARROS, I. Efeito da freqüência de capinas na produção do cafeeiro. Bragantia, v.55, p.317-324, 1996. 